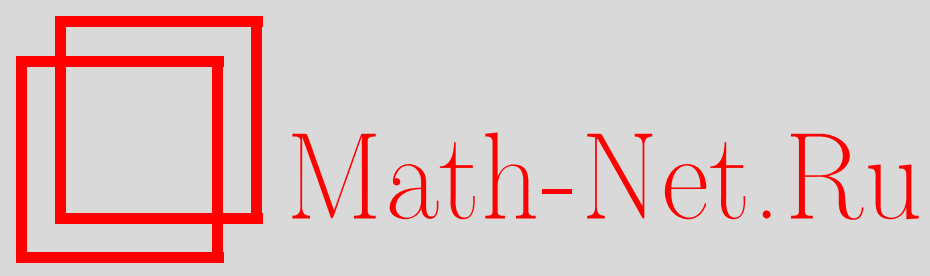

Л. П. Власов, Бикомпакт "две стрелки Александрова" и теория приближений, Матем. заметки, 2001, том 69, выпуск $6,820-827$

DOI: https://doi.org/10.4213/mzm696

Использование Общероссийского математического портала Math-Net.Ru подразумевает, что вы прочитали и согласны с пользовательским соглашением http://www.mathnet.ru/rus/agreement

Параметры загрузки:

IP : 54.80 .97 .219

26 апреля 2023 г., 13:17:49

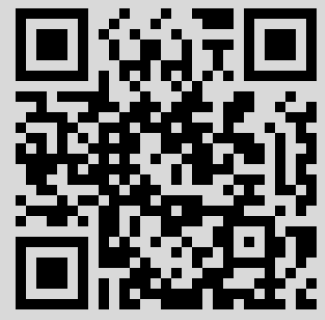




\section{БИКОМПАКТ “ДВЕ СТРЕЛКИ АЛЕКСАНДРОВА” И ТЕОРИЯ ПРИБЛИЖЕНИЙ}

\section{Л.П. Власов}

Построен бикомпакт $\widetilde{Q}$, аналогичный бикомпакту, известному как “две стрелки Александрова". Показано, что в вещественном пространстве $C(\widetilde{Q})$ не существует чебышевских подпространств конечной коразмерности $>1$, но они существуют в комплексном $C(\widetilde{Q})$.

Библиография: 10 названий.

Статья примыкает к исследованиям [1]-[6] и непосредственно продолжает работу автора [7].

Множество $M$ в нормированном пространстве $X$ назьвается чебышевским, если для любого $x \in X$ множество элементов наилучшего приближения

$$
P_{M} x=\{y \in M:\|x-y\|=d(x, M)\}
$$

одноточечно.

Вопрос о характеризации бикомпактов $Q$, для которых в $C(Q)$ существуют чебышевские подпространства конечной коразмерности, изучается с 60-х гг. Гаркави [2], [3] охарактеризовал компакты (метризуемые бикомпакты), для которых в вешественном пространстве $C(Q)$ сушествуют такие подпространства, и привел нетривиальные примеры бикомпактов, имеющих (и не имеюших) это свойство. Характеризацию бикомпактов осуществил Браун [4]. Ключевую роль при этом сьпрал бикомпакт “две стрелки Александрова", на котором возможно продолжение функции типа $(f(t)-z) /|f(t)-z|$ $(f \in C(Q), z \in \mathbb{R})$ с множества $\{t: f(t) \neq z\}$ до непрерьвной функции. В настоящей работе строится бикомпакт $\widetilde{Q}$ с таким же свойством в комплексном случае. Ранее в [7] автор доказал, что если в вешественном $C(Q)$ существуют чебьшевские подпространства коразмерности $n>1$, то они существуют и в комплексном $C(Q)$ для того же $Q$. В данной заметке устанавливается, что обратное утверждение неверно для $C(\widetilde{Q})$. Доказывается также теорема о характеризации бикомпактов $Q$, для которых в $C(Q)$ сушествуют чебьшевские подпространства конечной коразмерности $>1$.

В дальнейшем $Q$ - бесконечный бикомпакт, $C(Q)$ - комплексное [вешественное] пространство непрерывных комплексных [вещественных] функций на $Q$ с обычной нормой;

Работа выполнена при финансовой поддержке Российского фонда фундаментальных исследований, гранты № 96-01-00121 и № 99-01-00460. 
$C(Q)^{*}$ - пространство комплексных [вешественных] мер Радона на $Q,\|\mu\|=|\mu| Q=$ $\operatorname{var}(\mu, Q) ; S_{\mu}=\{t \in Q:|\mu| G>0$ для любого открытого $G \ni t\} ; Q^{\prime}[I]$ - множество предельных [изолированных] точек бикомпакта $Q ; \partial A$ - гранища, $A$ - внутренность, $|A|-$ мошность множества $A$.

Мы применяем следуюшие обозначения: $\mathbb{C}$ - поле комплексных чисел, $\mathbb{S} \subset \mathbb{C}$ - единичная окружность $|z|=1$. Через $\widetilde{\mathbb{C}}$ будем обозначать комплексную плоскость, дополненную "бесконечно удаленными точками" вида $\infty w=\infty \cdot w$, где $w \in \mathbb{S}$. (Символ $\infty$ означает $+\infty$ расширенной вешественной прямой.) Предполагается, что $z_{n} \rightarrow \infty w$, если $\left|z_{n}\right| \rightarrow \infty, z_{n} /\left|z_{n}\right| \rightarrow w, \infty w_{n} \rightarrow \infty w$ при $w_{n} \rightarrow w,(\infty w) \cdot 0=0, \infty \cdot 0=0$. (Равенства используются при интегрировании.) Пространство $\widetilde{\mathbb{C}}-$ бикомпактное расширение $\mathbb{C}-$ гомеоморфно “диску" $\mathbb{D}=\{z:|z| \leqslant 1\}$.

Нужные нам в дальнейшем свойства производной Радона-Никодима собраны в лемме 1 (см. [8, гл. III, п. 10], а также [7, лемма 1]).

Лемма 1. Пусть $Q$ - бикомпакт, $\mu, \nu, \lambda \in C(Q)^{*}, d \mu / d \nu=f$, m.e. $\mu e=\int_{e} f(t) d \nu$ для любого борелевского множества е $\subset Q$. Тогда $|\mu| e=\int_{e}|f(t)| d|\nu| ; d \nu / d \mu=1 / f$ на $\left\{t \in S_{\nu}: f(t) \neq 0\right\} ;$ если $d \nu / d \lambda=\varphi$, то $d \mu / d \lambda=d \mu / d \nu \cdot d \nu / d \lambda=f \varphi$, m.e. $\mu e=\int_{e} f d \nu=\int_{e} f \varphi d \lambda . \quad$ Если $h=d \mu / d|\mu|$, то $|h(t)|=1 \mu$-почти всюду. Если $g \in C(Q),\|g\| \leqslant 1$, то $|\mu| Q=\int_{Q} g(t) d \mu$ тогда и только тогда, когда $g=d|\mu| / d \mu$.

Нам понадобится ряд теорем, доказанных в [7].

Теорема 1 [7, теорема 2]. Для того чтобы подпространство L коразмерности п в комплексном или вещественном $C(Q)$ было чебышевским множеством, необходимо и достаточно, чтобы выполнялись условия:

(1а) для любой меры $\mu \in L^{\perp}$ существует непрерьвная производная Радона-Никодима $d \mu / d|\mu|$

(1б) $\left|Q \backslash S_{\mu}\right|<n$ для всех $\mu \in L^{\perp} \backslash\{0\}$;

(1в) любые меры $\mu, \nu \in L^{\perp} \backslash\{0\}$ взаимно абсолютно непрерывны на $Q^{\prime}$;

(1г) если $\left\{\mu_{1}, \mu_{2}, \ldots, \mu_{n}\right\}-$ базис в $L^{\perp}, I=Q \backslash Q^{\prime}=\left\{t_{1}, t_{2}, \ldots, t_{r}\right\}$ u $r<n$, mo $\operatorname{rank}\left\|\mu_{i}\left\{t_{k}\right\}\right\|_{k \leqslant r}^{i \leqslant n}=r$.

В вещественном случае аналогичную теорему ранее установил Гаркави [2], другие варианты см. также у Брауна [4].

СлЕДСТВИЕ 1. Если множество L чебышевское, то $Q=\bigcup_{\lambda \in L^{\perp}} S_{\lambda}$ и существует $\nu \in L^{\perp}$ c $S_{\nu}=Q$.

СЛЕДСТВИЕ 2. Для того чтобы подпространство

$$
L=\{\nu\}_{\perp}=\left\{x \in C(Q): \int_{Q} x d \nu=0\right\}
$$

коразмерности 1 в комплексном или вещественном $C(Q)$ было чебышевским множеством, необходимо и достаточно, чтобы существовала непрерывная производная Радона-Никодима $d \nu / d|\nu|$ и выполнялось условие $S_{\nu}=Q$.

Следуюшая теорема представляет собой незначительную переформулировку теоремы 5 из [7]. Здесь и далее используется обозначение

$$
N_{z, f}=\{t \in Q: f(t)=z\}, \quad z \in \mathbb{C}, \quad f \in C(Q, \widetilde{\mathbb{C}}) .
$$


Теорема 2 [7, теорема 5]. Пусть $\mu, \nu \in C(Q)^{*}$ линейно независимы $u S_{\nu}=Q$. Для того чтобы подпространство $L=\{\mu, \nu\}_{\perp}$ в комплексном или вещественном $C(Q)$ было чебьиевским множсеством, необходимо и достаточно, чтобы выполнялись условия:

(2а) существуют производнье Радона-Никодима $d \nu / d|\nu| \in C(Q), f=d \mu / d \nu \in$ $C(Q, \widetilde{\mathbb{C}})$

(2б) $\left|Q \backslash S_{\mu}\right| \leqslant 1$;

(2в) $\left|\stackrel{\circ}{*}_{z, f}\right| \leqslant 1 \partial \partial_{\text {я }}$ всех $z \in \mathbb{C}$;

(2г) $|\nu|\left(\partial N_{z, f}\right)=0$ для всех $z \in \mathbb{C}$;

(2д) для любого $z \in \mathbb{C}$ функиия $(f(t)-z) /|f(t)-z|$, определенная в точках $t \in Q \backslash N_{z, f}$, продолжсается до функиии $\psi_{z} \in C(Q)$.

Условие $S_{\nu}=Q$ не ограничивает общности в силу следствия 1 . В случае $f(t)=\infty w$ естественно считать, что $\psi_{z}(t)=(f(t)-z) /|f(t)-z|=w$, причем независимо от условия $\left(2\right.$ д) $\psi_{z}$ непрерывна на $Q \backslash N_{z, f}$, если $f \in C(Q, \widetilde{\mathbb{C}})$.

Теорема $3\left[7\right.$, теорема 6]. Пусть бикомпакт $Q$, мера $\nu \in C(Q)^{*}$ и функиия $f \in$ $C(Q, \widetilde{\mathbb{C}})$ таковы, ито

(3a) $S_{\nu}=Q$;

(3б) $d \nu / d|\nu| \in C(Q)$;

(3в) для любого $z \in \mathbb{C}$ мнохсество $N_{z, f}=I_{z} \cup B_{z}$, где $I_{z}=I \cap N_{z, f}$ пусто или состоит из одной точки, $a|\nu| B_{z}=0$ (это условие эквивалентно конъюнкции условий (2в) и $(2 г))$;

(Зг) для любого $z \in \mathbb{C}$ функиия $(f(t)-z) /|f(t)-z|$, определенная в точках $t \in Q \backslash N_{z, f}$, продолжается до функиии $\psi_{z} \in C(Q)$;

(3д) $\int_{Q}|f(t)|^{n-1} d|\nu|<\infty$.

Тогда если определить меры равенствами $\mu_{i} e=\int_{e} f(t)^{i-1} d \nu$ для любого борелевского мнохества е $(i=1, \ldots, n)$, то подпространство

$$
L=\left\{\mu_{1}, \mu_{2}, \ldots, \mu_{n}\right\}_{\perp}=\left\{x \in C(Q): \int_{Q} x d \mu_{i}=0 \quad \forall i=1, \ldots, n\right\}
$$

является чебышевским.

Определим бикомпакт $\widetilde{Q}$ следуюшим образом. Пусть

$$
A=\widetilde{\mathbb{C}} \backslash \mathbb{C}=\{\infty w: w \in \mathbb{S}\}, \quad \widetilde{Q}=(\mathbb{C} \times \mathbb{S}) \cup A, \quad \mathbb{C}_{s}=\mathbb{C} \times\{s\}, \quad s \in \mathbb{S} .
$$

Базу окрестностей точки $t \in \widetilde{Q}$ определим так: если $\varepsilon, \alpha>0$, то положим

$$
\begin{gathered}
O_{\varepsilon \alpha}\left(z_{0}, s_{0}\right)=\left\{z \in \mathbb{C}:\left|z-z_{0}\right|<\varepsilon,\left|\frac{z-z_{0}}{\left|z-z_{0}\right|}-s_{0}\right|<\alpha\right\}, \\
A_{\alpha}\left(z_{0}, s_{0}\right)=\left\{\left(z_{0}, s\right):\left|s-s_{0}\right|<\alpha\right\}, \\
O_{\varepsilon \alpha}(\infty w)=\left\{z \in \mathbb{C}:|z|>1 / \varepsilon,\left|\frac{z}{|z|}-w\right|<\alpha\right\}, \\
A_{\alpha}(\infty w)=\{\infty v: v \in \mathbb{S},|v-w|<\alpha\}, \\
W_{\varepsilon \alpha}(t)=\left(O_{\varepsilon \alpha}(t) \times \mathbb{S}\right) \cup A_{\alpha}(t) .
\end{gathered}
$$


Нетрудно проверить, что для любой точки $\tau \in W_{\varepsilon \alpha}(t)$ существуют $\varepsilon^{\prime}, \alpha^{\prime}$ такие, что $W_{\varepsilon^{\prime} \alpha^{\prime}}(\tau) \subset W_{\varepsilon \alpha}(t)$. Поэтому для любых $\varepsilon, \alpha, \varepsilon^{\prime}, \alpha^{\prime}>0, \tau \in W_{\varepsilon \alpha}(t) \cap W_{\varepsilon^{\prime} \alpha^{\prime}}(t)$ существуют $\varepsilon^{\prime \prime}, \alpha^{\prime \prime}$ такие, что $W_{\varepsilon^{\prime \prime} \alpha^{\prime \prime}}(\tau) \subset W_{\varepsilon \alpha}(t) \cap W_{\varepsilon^{\prime} \alpha^{\prime}}(t)$. В силу [9, глава 1 , теорема 11$]$ множества $W_{\varepsilon \alpha}(t), \varepsilon, \alpha>0, t \in \widetilde{Q}$, образуют базу открытых множеств. Поскольку $\varepsilon, \alpha$ можно брать рациональными, $\widetilde{Q}$ - пространство с первой аксиомой счетности. Следовательно, топология в $\widetilde{Q}$ определяется сходяшимися последовательностями [9, глава 2 , теорема 8]. Легко видеть, что

1) $t_{n}=\left(z_{n}, s_{n}\right) \rightarrow t_{0}=\left(z_{0}, s_{0}\right)$, если

$$
\begin{gathered}
z_{n} \rightarrow z_{0}, \quad \frac{z_{n}-z_{0}}{\left|z_{n}-z_{0}\right|} \rightarrow s_{0} \quad \text { в случае } z_{n} \neq z_{0}, \\
s_{n} \rightarrow s_{0} \quad \text { в случае } z_{n} \equiv z_{0}
\end{gathered}
$$

2) $\left(z_{n}, s_{n}\right) \rightarrow \infty w$, если

$$
\left|z_{n}\right| \rightarrow \infty, \quad \frac{z_{n}}{\left|z_{n}\right|} \rightarrow w
$$

3) $\infty w_{n} \rightarrow \infty w$, если $w_{n} \rightarrow w$.

Те же соотношения верны и для направленности $t_{\alpha}(n$ заменяется на $\alpha)$. В дальнейшем топологию $\widetilde{Q}$ будем обозначать через $\theta$. Через $\theta_{0}$ обозначим обычную топологию в $\mathbb{C}$, $\mathbb{S}$, топологию произведения $\mathbb{C} \times \mathbb{S}$ и топологию в $\widetilde{Q}$, совпадающую с $\theta_{0}$ в $\mathbb{C} \times \mathbb{S}$ и с $\theta$ в $A$.

Нетрудно показать, что $\widetilde{Q}$ хаусдорфово и что каждая направленность имеет $\theta$-сходящуюся поднаправленность, поэтому $\widetilde{Q}$ - бикомпакт.

Бикомпакт “две стрелки Александрова" топологически содержится в $\widetilde{Q}$. Например, множество

$$
\{(z, s): z \in[0,1), s=1\} \cup\{(z, s): z \in(0,1], s=-1\}
$$

гомеоморфно этому бикомпакту.

Лемма 2. Пространство $\widetilde{Q}-$ связный бикомпакт.

ДокАЗАТЕльство. Допустим, что существует открыто-замкнутое множество $F$ в $\widetilde{Q}$, непустое и отличное от $\widetilde{Q}$. Так как для любого $z \in \mathbb{C}$ топология $\theta$ совпадает с $\theta_{0}$ на $\{z\} \times \mathbb{S}$, все такие "окружности" $\theta$-связны. Отсюда $\bigcup_{z \in F}(\{z\} \times \mathbb{S}) \subset F$ и $\mathbb{C}_{s} \cap F \neq \varnothing$ для всех $s \in \mathbb{S}$. Тогда $\mathbb{C}_{s} \cap F \quad \theta_{0}$-замкнуто относительно $\mathbb{C}_{s}$ : если $\left(z_{n}, s\right) \in F$ и $\theta_{0}-\lim \left(z_{n}, s\right)=(z, s)$, то можно считать, что $\left(z_{n}-z\right) /\left|z_{n}-z\right| \rightarrow s_{0} \in \mathbb{S}$; поэтому $\theta-\lim \left(z_{n}, s\right)=\left(z, s_{0}\right)$, следовательно, $\left(z, s_{0}\right) \in F$ и по доказанному $(z, s) \in F$. Аналогично, $\mathbb{C}_{s} \cap(Q \backslash F) \theta_{0}$-замкнуто, что, очевидно, противоречит $\theta_{0}$-связности $\mathbb{C}_{s}$.

TЕОремА 4. В комплексном $C(\widetilde{Q})$ существуют чебышевские подпространства $L$ любой конечной коразмерности $n>1$. В вещественном $C(\widetilde{Q})$ таких подпространств не существует.

ДокАЗАТЕЛЬСтво. Воспользуемся теоремой 3. Пусть $m$ - мера Лебега на $\mathbb{S}, m_{2}-$ двумерная мера Лебега на $\mathbb{C}$. Чтобы построить меру $\nu$, рассмотрим положительную на $\mathbb{C}$ и быстро убывающую на бесконечности функцию $\rho$ такую, что

$$
\int_{\mathbb{C}}|z|^{n-1} \rho(z) d m_{2}(z)<\infty
$$


и положим $\nu_{1}(e)=\int_{e} \rho d m_{2}$ для любого борелевского множества $e \subset \mathbb{C}$. На $\widetilde{Q}$ зададим меру $\nu$, положив $|\nu| A=0, \nu=\nu_{1} \times m$ на $\mathbb{C} \times \mathbb{S}$. Тогда $\nu \in C(\widetilde{Q})^{*}, S_{\nu}=\widetilde{Q}$, $d \nu / d|\nu|=\mathbf{1} \in C(\widetilde{Q})$ (т.е. выполняются условия (3а), (3б)).

Зададим функцию $f$ :

$$
f(z, s)=z \quad \forall(z, s) \in \mathbb{C} \times \mathbb{S}, \quad f(\infty w)=\infty w .
$$

Нетрудно проверить, что $f \in C(\widetilde{Q}, \widetilde{\mathbb{C}})$. Далее, вьполняется условие $(3 в)$, ибо для любого $z \in \mathbb{C}$ вьполнены равенства

$$
N_{z, f}=\{t \in \widetilde{Q}: f(t)=z\}=\{z\} \times \mathbb{S}, \quad \nu\left(N_{z, f}\right)=\nu_{1}\{z\} \cdot m(\mathbb{S})=0
$$

Покажем, что вьполнено условие (3г). Найдем функцию $\psi_{z_{0}}\left(z_{0} \in \mathbb{C}\right)$ такую, что в точках множества $Q \backslash N_{z_{0}}$ она задается соотношением

$$
\psi_{z_{0}}(t)=\frac{f(t)-z_{0}}{\left|f(t)-z_{0}\right|}=\frac{z-z_{0}}{\left|z-z_{0}\right|}, \quad t=(z, s) \notin N_{z_{0}, f}, \quad \text { т.e. } z \neq z_{0} \text {. }
$$

Продолжая ее по непрерьвности, имеем

$$
\psi_{z_{0}}\left(z_{0}, s_{0}\right)=\lim _{(z, s) \rightarrow\left(z_{0}, s_{0}\right)} \psi_{z_{0}}(z, s)=\lim _{(z, s) \rightarrow\left(z_{0}, s_{0}\right)} \frac{z-z_{0}}{\left|z-z_{0}\right|}=s_{0} .
$$

Далее

$$
\psi_{z_{0}}(\infty w)=\infty w
$$

Непрерывность $\psi_{z_{0}}$ в точке $\infty w_{0}$ очевидна.

Покажем, что вьполнено условие (3д). Рассмотрим

$$
\begin{aligned}
\int_{\widetilde{Q}}|f(t)|^{n-1} d|\nu| & =\int_{\mathbb{C} \times \mathbb{S}}|f(z, s)|^{n-1} d\left(\nu_{1} \times m\right) \\
& =\int_{\mathbb{S}}\left\{\int_{\mathbb{C}}|f(z, s)|^{n-1} d \nu_{1}\right\} d m=\int_{\mathbb{C}}|z|^{n-1} d \nu_{1} \cdot m(\mathbb{S})<\infty .
\end{aligned}
$$

Таким образом, по теореме 3 в комплексном $C(\widetilde{Q})$ существуют подпространства конечной коразмерности $n>1$. Однако, в вещественном $C(Q)$ в случае связного $Q$ их, как показали Гаркави и Фелпс, не существует. В нашем изложении это можно получить из доказьваемой ниже теоремы 5.

Добавим, что в $C(\widetilde{Q})$ (и комплексном и вешественном) существуют чебьшевские подпространства коразмерности 1 , поскольку для $\nu$ вьполняются условия следствия 2 .

Дадим некоторую характеризацию бикомпактов $Q$, для которых комплексное (или вешественное) $C(Q)$ содержит чебьшевские подпространства конечной коразмерности. 
ТЕОРема 5. Пусть $Q$ - бикомпакт, $n>1$. Для того чтобы пространство $C(Q)$ (комплексное или вещественное) содержало чебышевское подпространство коразмерности $n$, необходимо и достаточно, чтобь существовали мера $\nu \in C(Q)^{*}$ и функиия $g \in C(Q)$, удовлетворяющие условиям

(5a) $S_{\nu}=Q$

(5б) $d \nu / d|\nu| \in C(Q)$;

(5в) для любого $z \in \mathbb{C}$ множество $N_{z, g}=I_{z} \cup B_{z}$, где $I_{z}=I \cap N_{z, g}$ пусто или состоит из одной точки, $a|\nu| B_{z}=0$;

(5г) для любого $z \in \mathbb{C}$ функиия $(g(t)-z) /|g(t)-z|$, определенная в точках $t \in Q \backslash N_{z, g}$, продолжается до функиии $\psi_{z} \in C(Q)$.

(Условия (5а) $-(5 г)$ не зависят от $n$.

ДокаЗАТЕльСтво. Необходимость. Вначале покажем, что для чебьшевского подпространства $L$ коразмерности $n$ сушествуют $\mu, \nu \in L^{\perp}$ такие, что $\{\mu, \nu\}_{\perp}-$ чебьшевское подпространство коразмерности 2 . Воспользуемся теоремой 1 . Пусть $r=\min \{n$, $|I|\} ;$ отметим, что $r \leqslant n$, а $I$ не более чем счетно. Возможны следуюшие два случая.

(I) $r=n \leqslant|I|$. Из пункта (1б) теоремы 1 следует, что для любых $t_{1}, t_{2}, \ldots, t_{n} \in I$ и для любого базиса $\mu_{1}, \mu_{2}, \ldots, \mu_{n}$ в $L^{\perp} \operatorname{det}\left\|\mu_{i} t_{k}\right\| \neq 0$. Возьмем $\nu \in L^{\perp}$ с $S_{\nu}=Q$ и любые $\tau_{i}, \tau_{j} \in I$. Пусть $M_{i j}$ - подпространство мер $\mu$ из $L^{\perp}$, пропорциональных $\nu$ на $\left\{\tau_{i}, \tau_{j}\right\}: \mu\left\{\tau_{i}\right\} / \nu\left\{\tau_{i}\right\}=\mu\left\{\tau_{j}\right\} / \nu\left\{\tau_{j}\right\}$. Из неравенства $\left|\mu\{\tau\}-\mu^{\prime}\{\tau\}\right| \leqslant\left\|\mu-\mu^{\prime}\right\|$ следует, что $M_{i j}$ замкнуто. Допустим, что $M_{i j}=L^{\perp}$. Это означает, что любая мера $\mu \in L^{\perp}$ пропорциональна $\nu$ на $\left\{\tau_{i}, \tau_{j}\right\}$. Пусть $\mu_{1}, \mu_{2}, \ldots, \mu_{n}$ - базис $L^{\perp}$, в котором $\mu_{2}=\nu$, а $t_{1}, t_{2}, \ldots, t_{n}$ - ряд изолированных точек, где $t_{1}=\tau_{i}, t_{2}=\tau_{j}$. Но тогда первые два столбца матрицы $\left\|\mu_{i} t_{k}\right\|$ линейно зависимы и $\operatorname{det}\left\|\mu_{i} t_{k}\right\|=0$, что противоречит сказанному вьше. Таким образом, $M_{i j}$ отлично от $L^{\perp}$. Тогда $\bigcup_{i, j} M_{i j}$ - множество первой категории в $L^{\perp}$. Следовательно, в $L^{\perp}$ найдется $\mu$, не пропорциональная $\nu$ ни на какой паре точек из $I$. Но это означает, что для $L^{\prime}=\{\mu, \nu\}_{\perp}$ вьполняется условие (1б) при $n=2$. По теореме $1 L^{\prime}$ - чебьшевское подпространство.

(II) $r=|I|<n ; I=\left\{t_{1}, t_{2}, \ldots, t_{r}\right\}$. По следствию 1 сушествует $\nu \in L^{\perp}$ с $S_{\nu}=Q$. Если $r=0$ или 1 , возьмем любую меру $\mu \in L^{\perp}$, линейно независимую с $\nu$. Тогда по теореме $1\{\mu, \nu\}_{\perp}$ - чебышевское подпространство. В общем случае из (1г) следует, что существуют меры $\mu_{1}, \mu_{2}, \ldots, \mu_{r} \in L^{\perp} \mathrm{c} \operatorname{det}\left\|\mu_{i} t_{k}\right\| \neq 0$. Поэтому сушествует $\mu \in L^{\perp}$ такая, что $\mu\left\{t_{i}\right\}=i \nu\left\{t_{i}\right\}(i=1,2, \ldots, r)$. Так как $\mu$ не пропорциональна $\nu$ ни на какой паре точек из $I$, это означает, что вьполняется условие (1б) при $n=2$. В силу $r \geqslant 2$ условие (1г) проверять не нужно, а условия (1а) и (1в) даны. Следовательно, $\{\mu, \nu\}_{\perp}-$ чебьшевское подпространство.

Заметим теперь, что по теореме 2 и следствию 1 определяются мера $\nu$, для которой выполняются условия (5a), (5б), и функция $f$, для которой (вместо функции $g$ ) выполняются (5в) и (5г). Покажем, что функцию $f \in C(Q, \widetilde{\mathbb{C}})$ можно заменить функцией $g(t)=f(t) /(1+|f(t)|)$, которая принадлежит $C(Q)$. Так как $g(t) /(1-|g(t)|)=f(t)$, то для $z^{\prime}$ с $\left|z^{\prime}\right|<1$ и $z=z^{\prime} /\left(1-\left|z^{\prime}\right|\right)$ имеем $N_{z^{\prime}, g}=N_{z, f}$, а для $\left|z^{\prime}\right| \geqslant 1 N_{z^{\prime}, g}=\varnothing$. Это дает (5в).

Докажем (5г) для $g$. Для этого нужно показать, что в каждой точке $t_{0} \in \partial N_{z^{\prime}, g}$ 
$\left(\left|z^{\prime}\right|<1\right)$ существует предел

$$
\lim \frac{g(t)-z^{\prime}}{\left|g(t)-z^{\prime}\right|}, \quad \text { где } t \rightarrow t_{0}, \quad t \notin N_{z^{\prime}, g},
$$

или, что то же ввиду $N_{z^{\prime}, g}=N_{z_{0}, f}, z_{0}=z^{\prime} /\left(1-\left|z^{\prime}\right|\right), f\left(t_{0}\right)=z_{0}$, предел

$$
\lim _{t \rightarrow t_{0}} \frac{D}{|D|}, \quad \text { где } \quad D=\frac{f(t)}{1+|f(t)|}-\frac{z_{0}}{1+\left|z_{0}\right|} \text {. }
$$

Полагая $f(t)=z$, мы видим, что $z \rightarrow z_{0}$. Далее, в силу условия (5г) для функции $f$ $\left(z-z_{0}\right) /\left|z-z_{0}\right| \rightarrow s_{0}$, где $s_{0} \in \mathbb{S}$. Обозначим через $B$ числитель дроби

$$
\frac{z-z_{0}-|z| z_{0}+z\left|z_{0}\right|}{\left.(1+|z|)\left(1+\mid z_{0}\right) \mid\right)}=\frac{z}{1+|z|}-\frac{z_{0}}{1+\left|z_{0}\right|} \text {. }
$$

Нам нужно доказать, что $B /|B|$ сходится, когда $z \rightarrow z_{0},\left(z-z_{0}\right) /\left|z-z_{0}\right| \rightarrow s_{0}$. Имеем

$$
\begin{aligned}
z \bar{z}-z_{0} \bar{z}_{0} & =z \bar{z}-z_{0} \bar{z}+z_{0} \bar{z}-z_{0} \bar{z}_{0}=\bar{z}\left(z-z_{0}\right)+z_{0}\left(\overline{z-z_{0}}\right) \\
& =\bar{z}\left(z-z_{0}\right)+z_{0}\left(z-z_{0}\right) \frac{\overline{z-z_{0}}}{z-z_{0}}=\left(z-z_{0}\right)\left[\bar{z}+z_{0}\left(\frac{\left|z-z_{0}\right|}{z-z_{0}}\right)^{2}\right] .
\end{aligned}
$$

Далее

$$
\begin{aligned}
B & =z-z_{0}-|z| z_{0}+z\left|z_{0}\right|-z_{0}\left|z_{0}\right|+z_{0}\left|z_{0}\right|=z-z_{0}+\left(z-z_{0}\right)\left|z_{0}\right|-\left(|z|-\left|z_{0}\right|\right) z_{0} \\
& =\left(z-z_{0}\right)\left(1+\left|z_{0}\right|\right)-\frac{z_{0}}{|z|+\left|z_{0}\right|}\left(z \bar{z}-z_{0} \bar{z}_{0}\right) \\
& =\left(z-z_{0}\right)\left[1+\left|z_{0}\right|-\frac{z_{0}}{|z|+\left|z_{0}\right|}\left(\bar{z}+\frac{z_{0}}{s^{2}}\right)\right],
\end{aligned}
$$

где $s=\left(z-z_{0}\right) /\left|z-z_{0}\right| \rightarrow s_{0}$. Обозначим выражение в квадратных скобках через $E$, тогда $B /|B|=s E /|E|, E$ равно 1 при $z_{0}=0$, а при $z_{0} \neq 0$ стремится к числу

$$
1+\left|z_{0}\right|-\frac{z_{0}}{2\left|z_{0}\right|}\left(\bar{z}_{0}+\frac{z_{0}}{s_{0}^{2}}\right)
$$

которое не равно 0, ибо

$$
1+\left|z_{0}\right|>\left|z_{0}\right| \geqslant\left|\frac{z_{0}}{2\left|z_{0}\right|}\left(\bar{z}_{0}+\frac{z_{0}}{s_{0}^{2}}\right)\right| .
$$

Таким образом, $B /|B|$ сходится и $g$ удовлетворяет всем условиям теоремы 3 .

Достаточность. В силу ограниченности функции $g$ она удовлетворяет всем условиям теоремы 3. Таким образом, если в теореме 3 функцию $f$ заменить на $g$, то получим чебьшевское подпространство $L$ коразмерности $n$.

Заметим, что в вещественном случае условия теоремы 5 по существу совпадают с условиями теоремы 2 Брауна [4]. Комплексньй аналог его теоремы 3 , даюший более глубокую характеризацию бикомпактов, нам получить не удалось.

Автор выражает благодарность Е. Г. Пыткееву, указавшему на сходство построения бикомпакта с общей конструкцией Федорчука [10], и Н. И. Черных за полезные замечания. 


\section{СПИСОК ЦИТИРОВАННОЙ ЛИТЕРАТУРЫ}

[1] Phelps R.R. C̆ebyšev subspaces of finite codimension in $C(X)$ // Pacific J. Math. 1963. V. 13. № 2. P. 647-655.

[2] Гаркави А. Л. Задача Хелли и наилучшее приближениев пространстве непрерьвных функций // Изв. АН СССР. Сер. матем. 1967. Т. 31. № 3. С. 641-656.

[3] Гаркави А. Л. О компактах, допускающих чебьшшевские системы мер // Матем. сб. 1967. T. 74(116). № 2. C. 209-217.

[4] Brown A. L. Chebyshev subspaces of finite codimension in spaces of continuous functions // J. Austral. Math. Soc. Ser. A. 1978. V. 26. № 1. P. 99-109.

[5] Власов Л. П. Аппроксимативные свойства подпространств конечной коразмерности в $C(Q)$ // Матем. заметки. 1980. Т. 28. № 2. С. 205-222.

[6] Власов Л. П. Сушествование элементов наилучшего приближения в комплексном $C(Q) / /$ Матем. заметки. 1986. Т. 40. № 5. С. 627-634.

[7] Власов Л. П. Чебышевские подпространства конечной коразмерности в комплексном $C(Q)$ // Матем. заметки. 1997. Т. 62. № 2. С. 178-191.

[8] Данфорд Н., Шварц Дж. Линейные операторы. Общая теория. ИЛ: М., 1962.

[9] Келли Дж. Л. Общая топология. М.: Наука, 1968.

[10] Федорчук В. О бикомпактах с несовпадающими размерностями // Докл. АН СССР. 1968. T. 182. № 2. C. $275-277$.

Институт математики и механики УрО РАН

Поступило

E-mail : leonid.vlasov@apprekat.imm.uran.ru 26.07.1999 